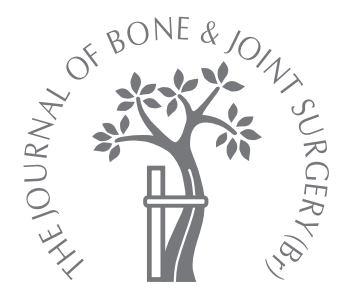

\title{
The factors affecting outcome after non- vascular bone grafting and internal fixation for nonunion of the scaphoid
}

C. Ramamurthy,

L. Cutler,

D. Nuttall,

A. J. M. Simison,

I. A. Trail,

J. K. Stanley

From the

Wrightington

Hospital, Appley

Bridge, England

In C. Ramamurthy, MB BS DOrth, MRCS, Registrar University Hospital of North Staffordshire, 578 Newcastle Road, Stoke-on-Trent, ST4 6QG, UK.

- L. Cutler, MB BS, FRCS Ed, FRCS (Trauma and Orth), Consultant Orthopaedic Surgeon

Leicester Royal Infirmary, Infirmary Square, Leicester LE1 5WW, UK.

A J. M. Simison, FRCS Ed(Orth), Mch Orth, Consultant Orthopaedic Surgeon Arrowe Park Hospital, Arrowe Park Road,

Upton, Wirral CH49 5PE, UK.

D. Nuttall, PhD, Research Fellow

I. A. Trail, MD, FRCS, Consultant Upper Limb Surgeon

J. K. Stanley, FRCS, FRCSE, Mch Orth, Professor of Hand Surgery

Wrightington Hospital, Hall Lane, Appley Bridge WN6 9EP, UK.

Correspondence should be sent to $\mathrm{Mr}$ C. Ramamurthy; e-mail: ramac@myway.com

(C)2007 British Editorial Society of Bone and Joint Surgery doi:10.1302/0301-620X.89B5 $18183 \$ 2.00$

$J$ Bone Joint Surg [Br] 2007;89-B:627-32. Received 25 May 2006; Accepted after revision 6 February 2007

\begin{abstract}
This study identified variables which influence the outcome of surgical management on 126 ununited scaphoid fractures managed by internal fixation and non-vascular bone grafting. The site of fracture was defined by a new method: the ratio of the length of the proximal fragment to the sum of the lengths of both fragments, calculated using specific views in the plain radiographs. Bone healing occurred in $71 \%(89)$ of cases. Only the site of nonunion (p $\left.=1 \times 10^{-6}\right)$ and the delay to surgery $(p=0.001)$ remained significant on multivariate analysis. The effect of surgical delay on the probability of union increased as the fracture site moved proximally. A prediction model was produced by stepwise logistic regression analysis, enabling the surgeon to predict the success of surgery where the site of the nonunion and delay to surgery is known.
\end{abstract}

Although much has been written about nonunion of the scaphoid, its management remains difficult and controversial. Accurate classification is important to determine treatment and predict outcome. The systems described in the literature are based on the site of fracture, ${ }^{1-4}$ the stability of the nonunion, ${ }^{5}$ the intra-operative characteristics of the site of nonunion ${ }^{6}$ and on the presence or absence of arthritis in the adjacent joints. ${ }^{7,8}$

The site of a scaphoid fracture can be described using several methods. ${ }^{1-4}$ The most commonly used divides the scaphoid into thirds. The drawback of this method that the division is arbitrary as there are no bony landmarks on the scaphoid bone allowing accurate division into thirds. In addition, the length of the scaphoid can appear different in different views and as such any fracture could easily be misclassified using the equal third method. ${ }^{9}$ The characteristics of nonunions of the proximal and distal thirds are entirely different and those at the waist can overlap these sites. The site of the nonunion is a continuous variable, and a classification system which respects this may give a clearer insight into the behaviour of scaphoid fractures.

Bony consolidation of an established scaphoid nonunion may be achieved by operation, but no method is effective in every patient. The rates of success in published series range between $65 \%$ and $95 \% .^{10-18}$ These differences may reflect varying surgical techniques and differences in the case mix. Many factors may affect the probability of achieving union. ${ }^{10-18}$ When many variables are present, the simple statistical analysis of one variable against the outcome may give misleading results, especially in a small series. Multivariate analysis explores relationships between many variables, but it has rarely been applied to the study of scaphoid nonunion. ${ }^{14}$ If the outcome of treatment is influenced by several variables, the mean rate of success may be a poor indicator of the optimum treatment in an individual patient and the likely outcome. Additionally, if the outcome of treatment is not supported by a definition of the case mix, where all the variables are taken into account, it will provide a poor means of comparing different studies. A model that could predict the outcome through its consideration of the most important variables would be a useful tool, but this does require inclusion of a large number of cases in the study.

This study describes a new way of defining the site of fracture in the scaphoid that is both reproducible, easy to use in routine clinical practice and that predicts the outcome of surgery for nonunion using methods of logistic regression.

\section{Patients and Methods}

A retrospective review of case records identified 159 patients in whom established nonunion of the scaphoid had been treated by bone grafting and internal fixation. There were 107 patients treated at Wrightington Hospital and 52 patients at Arrowe Park Hospital, between April 1991 and February 2003 and between April 1996 and November 2002, 
Table I. Pattern of presentation of nonunion of the scaphoid

\begin{tabular}{lll}
\hline Patient group & Pattern of presentation & Number of patients \\
\hline 1 & $\begin{array}{l}\text { Patients with a scaphoid fracture treated in a cast and followed up ade- } \\
\text { quately } \\
\text { Patients with a scaphoid fracture treated in a cast and followed up inade- } \\
\text { quately }\end{array}$ & $\begin{array}{l}37 \\
\text { Patients with a scaphoid fracture and associated perilunate dislocation or } \\
\text { fracture of the distal radius, who had immediate surgical fixation } \\
\text { Patients who were never treated at the time of initial injury but presented } \\
\text { with persisting symptoms following re-injury }\end{array}$ \\
4 & Patients who had previous unsuccessful surgery for nonunion & 6 \\
5 & & 6
\end{tabular}

Table II. Demographic details (based on number of nonunions)

\begin{tabular}{lc}
\hline & Value \\
\hline Mean age in years (range) & 28 (9 to 59) \\
Gender & 119 \\
$\quad$ Male & 7 \\
$\quad$ Female & 62 \\
Hand dominance & 64 \\
$\quad$ Dominant & 4.5 (0.25 to 16) \\
$\quad$ Non-dominant & 56 \\
Mean time interval from injury to surgery (yrs; & \\
range) & 21 \\
DISI* (radiolunate angle $>10^{\circ}$ ) & 4 \\
Osteoarthritis & \\
$\quad$ Grade I & 103 \\
$\quad$ Grade II & 11 \\
Fracture pattern & 12 \\
$\quad$ Transverse & \\
$\quad$ Vertically oblique & \\
$\quad$ Horizontally oblique & 93 \\
Displacement at fracture site & 33 \\
$\quad$ Displaced (> 2 mm) & \\
$\quad$ Undisplaced & *DISI, dorsal intercalated segment instability
\end{tabular}

respectively. Nonunion was defined as persistence of a fracture gap at least three months after the initial trauma, with associated resorption of bone and cystic changes at the fracture site, as seen on the radiographs. ${ }^{19}$ There were five different patterns of presentation (Table I).

Patients were included in the study if they had established nonunion of the scaphoid and had undergone nonvascular bone grafting and internal fixation, either with a screw or Kirschner (K)-wires. A total of 19 patients could not remember the month and year of the initial injury, a further nine had less than six months follow-up, and six had undergone previous failed surgery for established nonunion. They were thus excluded from the study, as was one patient with a fracture in the coronal plane, leaving 124 patients (126 nonunions) in the study (Table II). The operations were performed by one of the three senior authors (AJMS, IAT, JKS). A dorsal approach was used in 19 patients with fractures involving the proximal pole. In the remainder, the fracture was approached through an anterior incision. Fibrous tissue between the fragments was excised, and the fracture surfaces were curetted. If dorsal intercalated segment instability was present, a K-wire was used to correct the deformity. The bone graft was obtained from the iliac crest in 70 patients and from the distal radius in 54. Corticocancellous bone grafts were used when the bone defect was large, and multiple cancellous bone chips when the defect was small. Internal fixation was accomplished using two $1.6 \mathrm{~mm} \mathrm{~K}$-wires in 46 nonunions and a single screw in 80 cases. Herbert (Zimmer, Warsaw, Indiana), $3.5 \mathrm{~mm}$ (Synthes, Waldenburg, Switzerland) AO and Acutrak screws (Acumed, Beaverton, Oregon) were used for fixation in 57, 15 and eight cases, respectively. The wrist was immobilised for 8 to 12 weeks after operation. All the patients were followed up clinically and radiologically by one of the three operating surgeons at six and 12 weeks, and six months, or until union was achieved.

The fracture site was described by the fragment ratio, measured using plain radiographs. Three radiographs were converted to digital images using a Nikon Coolpix 5000 digital camera (Nikon Corp., Tokyo, Japan) with standard settings. In the digital format, it was easier to identify the margins of the fragments and to make accurate measurements of their size. As no single view enables visualisation of the fracture site in all cases, the long axis of the scaphoid and the fracture are best visualised ${ }^{20,21}$ in the ulnardeviated and posteroanterior (PA) semi-pronated oblique views. In order to calculate the amount of projectional error causing variation in measurement between these two views, the whole length of the scaphoid was measured in 138 films of normal scaphoids, taken consecutively, with standard magnification settings. The estimated standard deviation (SD) within subjects was $0.58 \mathrm{~mm}$ and the intraclass correlation coefficient was 0.932 . The repeatability index of $1.6 \mathrm{~mm}$ is equivalent to $6.7 \%$ of the mean length of the scaphoid. As the variation in the measured length was not significant, these two views were used to calculate the fragment ratio. The ulnar-deviated posteroanterior (PA) view was used in 67 nonunions, and the semi-pronated oblique view in 59 .

The bone fragments were measured (Fig. 1) using the measurement tool in Adobe Photoshop version 6.0 (Adobe Systems Inc., San Jose, California). Horizontal lines were drawn at the ends of each fragment to define the length of the fragment. Its middle was identified and a line was drawn between these points to connect the horizontal lines. The length of this line was measured to determine the size of the fragment. The fragment ratio was then calculated by 


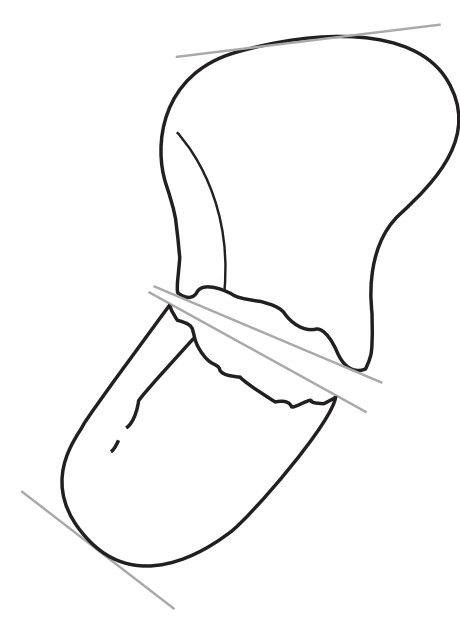

Fig. 1a

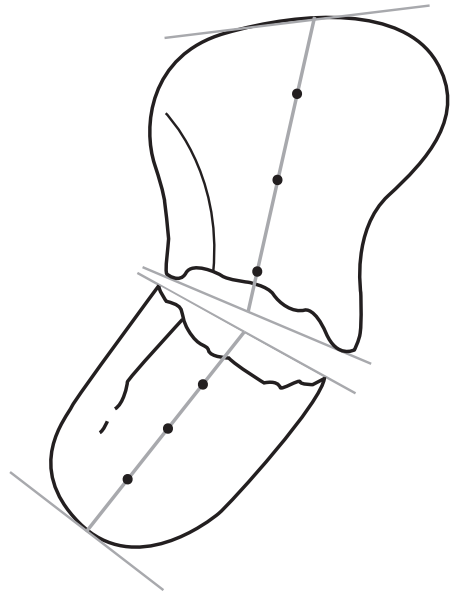

Fig. 1b

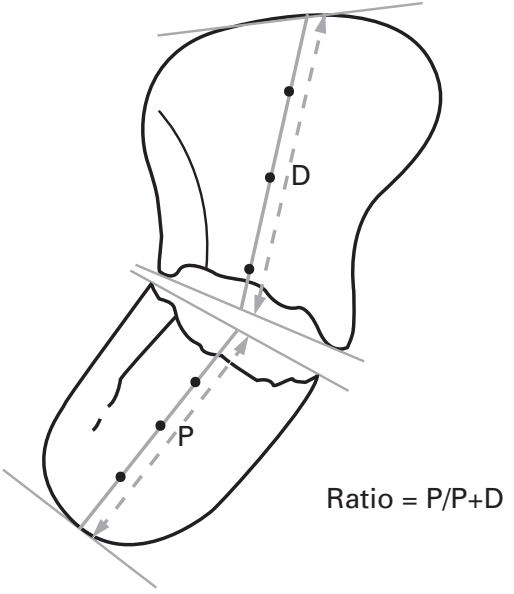

Fig. 1c

Illustrations defining the fracture site by the fragment ratio. a) Horizontal lines were drawn to define the extent of the fragment, b) the middle of the fragment was identified and a midline was drawn connecting the two horizontal lines, $c$ ) the lengths of these lines ( $P, D)$ were measured to determine the fragment size ( $P$, proximal fragment; $D$, distal fragment).

dividing the proximal fragment size by the sum of the sizes of the proximal and distal fragments. This value was used to describe the site of nonunion. In the 11 vertically oblique fractures, the same procedure was performed but because the line is drawn along the middle of a fragment, it measured the mean length of both.

The outcome variable was defined as bony union or persistent nonunion. Although CT and MRI scans are more reliable for assessing bony union, serial radiographs taken at follow-up were used to assess union, as described by Dias, ${ }^{22}$ because of the retrospective nature of this study. Bony union was defined as disappearance of the nonunion gap, absence of loosening of the internal fixation and no displacement of the fragment or graft. Impending nonunion was determined by a persistent gap, loosening of the fixation or displacement of the fragment. Persistent nonunion was defined when the radiological appearances suggested that the fracture had not united and would not do so without further intervention. The outcome variable was defined as bony union or persistent nonunion.

Statistical analysis. Intra-observer variability in defining the fracture site was assessed. Three independent blinded observers (CR, LC, DN) were asked to measure the fragment ratios for all 126 nonunions. An intraclass correlation coefficient and a repeatability coefficient were calculated in Statsdirect 2.4.4 (Stats Direct Ltd, Cheshire, England) ${ }^{23}$ using the Bland Altman method. ${ }^{24}$ Univariate logistic regression analyses were carried out using each of 15 possible explanatory variables: age, gender, hand dominance, the site of nonunion, the fracture pattern, displacement at the nonunion site, the presence of dorsal intercalated segment instability, the presence of osteoarthritis, previous surgical fixation of an acute fracture, the time interval to surgery, the surgeon, smoking, initial conservative treatment, the method of fixation and the type of graft. Data on smoking was only recorded in 94 patients. We did not examine vascularity, because we believe that its assessment is both subjective and controversial. All logistic regression analyses were performed using SPSS 10.1 (statistical package for the Social Sciences, SPSS Inc., Chicago, Illinois) with significance being achieved if $\mathrm{p}<0.05$ in all instances.

Multivariate logistic regression analyses were also performed. All 15 explanatory variables were initially included, and a stepwise procedure was used to find a subset of variables that could be combined in a model to predict union. The model's performance in predicting the probability of union was measured using receiver operating characteristic (ROC) analysis ${ }^{25}$ in Statsdirect. The area under the curve was used as a measure of predicting the probability of union, where it ranges from 0.5 (no better than chance) to 1.0 (perfection), as well as the sensitivity, the true positive rate of union and specificity, and the true negative rate of union.

\section{Results}

The mean period of follow-up was 12.9 months (6 to 38 ). Out of 126 nonunions, $90(71 \%)$ showed union in the radiographs taken at follow-up. In $28(22 \%)$ there was persistent nonunion and eight $(6 \%)$ showed impending nonunion, ${ }^{22}$ which were taken as failures. Of the 36 failures, 15 $(42 \%)$ were asymptomatic and $21(58 \%)$ were symptomatic. Out of 21 symptomatic nonunions 13 had further surgery. The distribution of fractures by site is shown in Table III. The rates of union varied from $27 \%$ for fractures at the proximal pole to $100 \%$ for those in the distal third (Fig. 2). 
Table III. Distribution of the fractures together with the rate of union by fracture site as determined by the fragment ratio method of classification

\begin{tabular}{lcc}
\hline Fracture site & Number of fractures & Number united (\%) \\
\hline 0.15 to 0.30 & 15 & $4(27)$ \\
0.31 to 0.45 & 33 & $19(58)$ \\
0.46 to 0.60 & 48 & $40(83)$ \\
0.61 to 0.75 & 30 & $27(90)$ \\
\hline
\end{tabular}
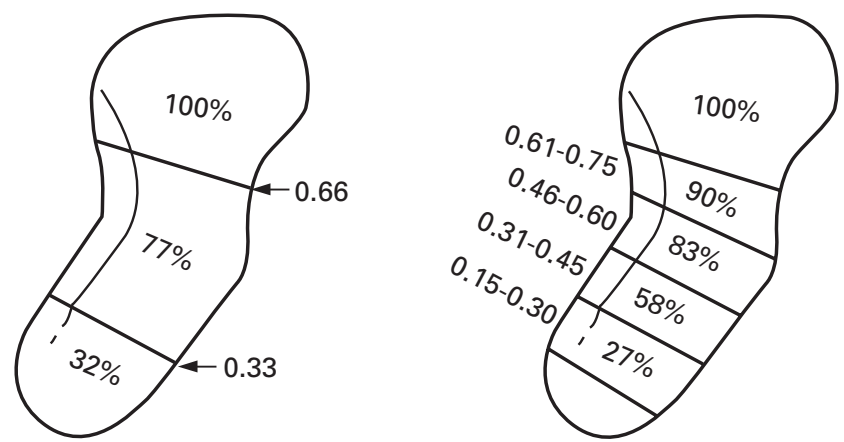

Fig. 2

Illustrations to show the rate of union in the a) equal third classification and b) fragment ratio method.

Repeatability of the technique in measuring the fragment ratio gave an estimated intraclass correlation coefficient of 0.895 and a within-subjects SD of 0.039 for the three observers, with a repeatability index of 0.108 at the $5 \%$ level. Univariate logistical analysis of all the fractures revealed that the site of nonunion $\left(\mathrm{p}=1 \times 10^{-6}\right)$, the time between injury and surgery $(\mathrm{p}=0.018)$ and the method of fixation $(\mathrm{p}=0.049)$ were statistically significant factors (Table IV). Patients who had screw fixation had, approximately, twice as much chance of going on to union as those with K-wire fixation. However, the $95 \%$ confidence interval $(\mathrm{CI})$ is wide and the difference may actually be as low as $\times 1.004$. Factors determining the stability of the fracture pattern, displacement at the site of nonunion and the presence of dorsal intercalated segment instability were not significant. The other factors comprising age, gender, hand dominance, the presence of osteoarthritis, previous failed fixation of an acute fracture, initial conservative treatment, the surgeon and the type of graft used, had no significant effect on the outcome. The effect of smoking analysed in 94 patients had no significant influence on the rate of union.

Using the stepwise multivariate logistic regression analysis, ${ }^{26}$ only the site of the fracture and the time between injury and surgery were found to be significant $\left(\mathrm{p}=1 \times 10^{-6}\right)$. A predictive logistic regression model was created using the fragment ratio and time to surgery (Fig. 3 ). The more proximal the fracture, the lower the probability of union, and the longer the delay before surgery the lower the probability of union. For example, if the fragment ratio is 0.6 then the probabilities of a successful outcome are $98 \%$ and $81 \%$ for surgery carried out at one year and ten years, respectively. If surgery is carried out at one year a fracture with a fragment ratio of 0.4 has a probability of union of $83 \%$, but this falls to $27 \%$ if there is a delay of ten years.

Analysis of the receiver operating characteristic curve of the predicted probability of union revealed an area under

Table IV. Univariate logistic regression analysis with binary response variable: bony union/nonunion

\begin{tabular}{|c|c|c|c|c|}
\hline Explanatory value & Code & Exponent (odds ratio) & $95 \%$ confidence interval & p-value \\
\hline Dominance & No $=0$, Yes $=1$ & 0.96 & 0.44 to 2.07 & 0.91 \\
\hline Previous surgical fixation & $\mathrm{No}=0, \mathrm{Yes}=1$ & 0.89 & 0.25 to 3.09 & 0.85 \\
\hline Fracture pattern & Transverse $=0$, Oblique $=1$ & 1.16 & 0.42 to 3.24 & 0.77 \\
\hline Gender & Male $=0$, Female $=1$ & 0.001 & $0->10^{6}$ & 0.75 \\
\hline Type of graft & Iliac crest $=0$, distal radius $=1$ & 1.50 & 0.34 to 6.56 & 0.59 \\
\hline \multicolumn{5}{|l|}{ Surgeon } \\
\hline & 1 & & & 0.74 \\
\hline & 2 & 0.76 & 0.31 to 1.90 & 0.57 \\
\hline & 3 & 0.70 & 0.26 to 1.87 & 0.48 \\
\hline Displacement & $\mathrm{No}=0, \mathrm{Yes}=1$ & 1.35 & 0.54 to 3.35 & 0.52 \\
\hline Age & $\mathrm{No}=0, \mathrm{Yes}=1$ & 1.01 & 0.97 to 1.06 & 0.26 \\
\hline Conservative treatment & No $=0$, Yes $=1$ & 1.73 & 0.75 to 4.03 & 0.20 \\
\hline DISI $^{*}$ & $\mathrm{No}=0, \mathrm{Yes}=1$ & 0.59 & 0.29 to 1.29 & 0.18 \\
\hline Osteoarthritis & $\mathrm{No}=0, \mathrm{Yes}=1$ & 0.52 & 0.21 to 1.3 & 0.16 \\
\hline Smoking $^{\dagger}$ & $\mathrm{No}=0, \mathrm{Yes}=1$ & 0.49 & 0.2 to 1.22 & 0.13 \\
\hline Fixation & $\mathrm{K}$ wire $=0$, Screw $=1$ & 2.21 & 1.004 to 4.89 & 0.049 \\
\hline Time delay (yrs) & & 0.88 & 0.80 to 0.98 & 0.018 \\
\hline P/PD ratio $\neq$ & & 5653 & 57 to 204017 & $1 \times 10^{-6}$ \\
\hline
\end{tabular}




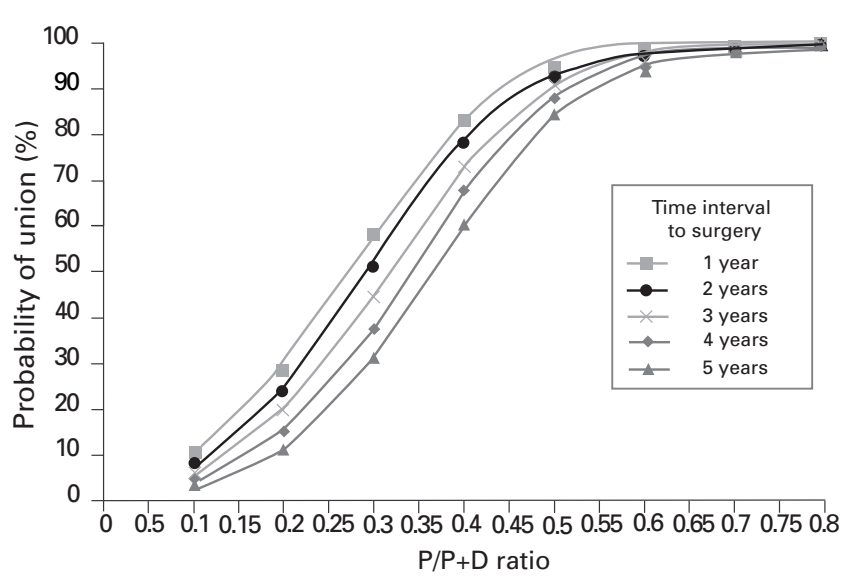

Fig. 3

Line graph showing multivariate logistic regression model with binary response variable: scaphoid bony union/nonunion of the scaphoid. Bony union $=12.275$ (proximal fragment length $/$ proximal fragment + distal fragment length) - 0.286 Delay - 3.066

the curve of $0.825(95 \% \mathrm{CI}, 0.74 \%$ to $0.91 \%)$, with a sensitivity (true positive rate) of $79 \%$ (95\% CI, 69\% to $87 \%)$ and a specificity (true negative rate) of $72 \%(95 \% \mathrm{CI}$, $55 \%$ to $86 \%$ ). The likelihood ratio (positive test) is 2.8 i.e. the model is likely to predict union at least 2.8 times as often for scaphoids that unite as for those which do not. The likelihood ratio (negative test) is 0.29 , indicating that approximately three in ten of the nonunions predicted by the model will actually unite.

\section{Discussion}

Although many studies in the past have highlighted the drawbacks of using plain radiography in the management of fractures of the scaphoid, ${ }^{21,27-29}$ it remains the most commonly used technique of imaging. When looking at the reproducibility of the radiological features of an acute fracture of the scaphoid in the study undertaken by Desai et $\mathrm{al}^{29}$ identification of the level of the fracture had the highest intra- or interobserver reproducibility compared with classifications of displacement and comminution. As the fragment ratio method involves identification of the fracture line in nonunion, where the fracture gap is better demarcated and simply measures the size of the fragments in two specific views, it should have better reproducibility than other methods of classification.

Describing the fracture site by the fragment ratio has a considerable advantage. It describes the site of the fracture more adequately and can therefore be used to improve the validity of comparisons between studies. Figure 2 shows that the prognosis for fractures in the middle third of the scaphoid differs significantly depending on whether the fracture is in the distal or proximal half of that third. At times when measurement may be imprecise, small variations in the radiological projection of the scaphoid may introduce errors, resulting in the fracture being classified into the wrong third of the scaphoid. However, the fragment ratio method would lead only to small errors. Moreover, a numerical value of the fragment ratio with time delay to surgery allows graphical illustration of the prediction of the success of treatment. This is easier to interpret in clinical practice.

In assessing the effects of multiple variables on the outcome of any process, it is necessary to use statistical methods that recognise specific effects and exclude confounding factors and co-linearity between variables. Few studies include both a large number of cases and a rigorous analysis of factors which may influence the success of surgery in fractures of the scaphoid. ${ }^{14}$ The overall rate of union in our study was $71 \%$, similar to other large series. ${ }^{5,6,10,14,30}$ Statistical analysis showed that the most important factors in predicting the outcome included the site of nonunion and the time between the initial trauma and surgery.

Pechlaner et $\mathrm{al}^{30}$ reviewed 197 nonunions that were treated by Russe grafting and concluded that the time between injury and operation is an important determinant of outcome. This factor is important whatever the method of treatment. ${ }^{17,30,31}$ In a study describing nonunion in terms of characteristics noted intra-operatively, the mean times between injury and surgery for fibrous union (D1), pseudarthrosis (D2) and sclerotic pseudarthrosis (D3) were 8.5 months (7 weeks to 7 years), 25.3 months (7 weeks to 20 years) and 52.4 months ( 3 months to 25 years), respectively. The mean rates of union in D1, D2 and D3 were $88 \%$ (65 of 74 cases), $66 \%$ (73 of 110 cases) and 50\% (25 of 50 cases), respectively. ${ }^{6}$ These findings suggest that the time delay influences both the changes occurring at the site of nonunion as well as its potential for healing when treated surgically.

Several studies have shown that fixation with a screw produces better results that with K-wires. ${ }^{11,32-34}$ Univariate logistic regression analysis supported this conclusion in our patients, but multivariate analysis indicated that only the site and age of the fracture were significant determinants of outcome. This implies that once adjustment had been made for the other variables in the model, fixation was not significant. Bone grafts harvested from the iliac crest and from the distal radius were equally effective, as noted by Schuind et al. ${ }^{14}$

An analysis of the pattern of presentation of scaphoid nonunion performed in an acute hospital, revealed that $77 \%$ (23 of 30) of the patients presented immediately following initial injury. ${ }^{35}$ In our study in a specialist hand unit, only $41 \%$ (65 of 159) of the patients presented soon after the injury. This allowed an analysis of a wide spectrum of patients, with a mean delay to treatment of 42 months ( 3 months to 16 years). As the time delay to surgery has a direct effect on the outcome, the pattern of presentation can influence the overall rate of union in a given series of patients. This could also explain the difference in the rates achieved in several studies performed in the past. 
There are four limitations of our study. It is retrospective, and if patients cannot remember when they injured their wrist, the prediction model cannot be used. If the fracture is in the coronal plane, the fragment ratio cannot be calculated, but this is rare. ${ }^{36}$ Finally, there are times when measurement of the size of the fragment may be less accurate because of variations in radiological projections. This error should be reduced if the fragment ratio method is used.

We have described a method of defining the fracture site that is practical, respects the continuously variable nature of this observation and appears less open to error than the traditional system. We have exposed differences in the behaviour of fractures within the 'middle third'. Our system offers a more valid method of defining case mix when comparing different studies. We have also confirmed the findings from previous studies stating that the site and age of the fracture are important determinants of outcome. The age of the fracture has a large effect in proximal but only a weak effect in distal fractures. This is of practical importance when deciding how to treat these injuries. We have developed a model that allows the probability of successful treatment to be determined when the interval between injury and surgery is known and the fracture site is described using the fragment ratio. The prognosis may differ considerably from that predicted on the basis of the overall success rate for this operation. By way of example, for a nonunion of the proximal third of the scaphoid which has been present for more than five years, the chance of achieving union with a non-vascular bone graft and screw fixation is only $30 \%$.

No benefits in any form have been received or will be received from a commercial party related directly or indirectly to the subject of this article.

\section{References}

1. Russe $\mathbf{0}$. Fracture of the carpal navicular: diagnosis, nonoperative treatment, and operative treatment. J Bone Joint Surg [Am] 1960;42-A:759-68.

2. Schernberg F, Elzein F, Gerard Y. Anatomo-radiological study of fractures of the carpal scaphoid bone: problems of abnormal callus. Rev Chir Orthop Reparatrice Appar Mot 1984;70 (Suppl 2):55-63 (in French).

3. Herbert TJ, Fisher WE. Management of the fractured scaphoid using a new bone screw. J Bone Joint Surg [Br] 1984;66-B:114-23.

4. Compson JP. The anatomy of acute scaphoid fractures: a three-dimensional analysis of patterns. J Bone Joint Surg [Br] 1998;80-B:218-24.

5. Cooney WP, Dobyns JH, Linscheid RL. Fractures of the scaphoid: a rational approach to management. Clin Orthop 1980;149:90-7

6. Filan SL, Herbert TJ. Herbert screw fixation of scaphoid fractures. J Bone Joint Surg [Br] 1996;78-B:519-29.

7. Hooning van Duyvenbode JFF, Keijser LCM, Hauet EJ, Obermann WR, Rozing PM. Pseudarthrosis of the scaphoid treated by the Matti-Russe operation: a longterm review of 77 cases. J Bone Joint Surg [Br] 1991;73-B:603-6.

8. Herndon JH. Scaphoid fractures and complications. Rosemont: American Academy of Orthopaedic Surgeons, 1994
9. Desai VV, Davis TR, Barton NJ. The prognostic value and reproducibility of the radiological features of the fractured scaphoid. J Hand Surg [Br] 1999;24:586-90.

10. Barton NJ. Experience with scaphoid grafting. J Hand Surg [Br] 1997;22:153-60.

11. Merrell GA, Wolfe SW, Slade JF 3rd. Treatment of scaphoid non-unions: quantitative meta-analysis of the literature. J Hand Surg [Am] 2002;27:685-91.

12. Muhnk B, Larsen CF. Bone grafting the scaphoid non-union: a systematic review of 147 publications including 5,246 cases of scaphoid non-union. Acta Orthop Scand 2004;75:618-29.

13. Nakamura R, Horii E, Watanabe K, Tsunoda K, Miura T. Scaphoid nonunion: factors affecting the functional outcome of open reduction and wedge grafting with Herbert screw fixation. J Hand Surg [Br] 1993;18:219-24.

14. Schuind F, Haentjens $\mathbf{P}$, Van Innis F, et al. Prognostic factors in the treatment of carpal scaphoid non-unions. J Hand Surg [Am] 1999;24:761-76.

15. Green DP. The effect of avascular necrosis on Russe bone grafting for scaphoid nonunion. J Hand Surg [Am] 1985;10:597-605.

16. Inoue G, Shionoya K, Kuwahata Y. Herbert screw fixation for scaphoid non-unions: an analysis of factors influencing outcome. Clin Orthop 1997;343:99-106.

17. Shah J, Jones WA. Factors affecting the outcome in 50 cases of scaphoid non-union treated with Herbert screw fixation. J Hand Surg [Br] 1998;23:680-5.

18. Trezies AJH, Davis TRC, Barton NJ. Factors influencing the outcome of bone grafting surgery for scaphoid fracture non-union. Injury 2000;31:605-7.

19. Simonian PT, Trumble TE. Scaphoid non-union. J Am Acad Orthop Surg 1994;2:18591.

20. Leslie IJ, Dickson RA. The fractured carpal scaphoid: natural history and factors influencing outcome. J Bone Joint Surg [Br] 1981;63-B:225-30.

21. Compson JP, Waterman JK, Heatley FW. The radiological anatomy of the scaphoid. Part 2: radiology. J Hand Surg [Br] 1997;22:8-15.

22. Dias JJ. Definition of union after acute fracture and surgery for fracture non-union of the scaphoid. J Hand Surg [Br] 2001;26:321-5.

23. Buchan IE. Statsdirect 2.4.4. http://www.statsdirect.com (date last accessed 14 May 2006).

24. Bland MJ, Altman DG. Measurement error. BMJ 1996;312:1654

25. Armitage P, Berry G. Statistical methods in medical research. Oxford: Blackwell Scientific Publications, 1994.

26. Tabachnick BG, Fidell LS. Using multivariate statistics. Third ed. New York: Harper Collins 1996.

27. Dias JJ, Taylor M, Thompson J, Brenkel IJ, Gregg PJ. Radiological signs of scaphoid fractures: an analysis of inter-observer agreement and reproducibility. $J$ Bone Joint Surg [Br] 1988;70-B:299-301.

28. Dias JJ, Brenkel IJ, Finlay DB. Patterns of union in fractures of the waist of the scaphoid. J Bone Joint Surg [Br] 1989;71-B:307-10.

29. Desai VV, Davis TR, Barton NJ. The prognostic value and reproducibility of the radiological features of the fractured scaphoid. J Hand Surg [Br] 1999;24:586-90.

30. Pechlaner S, Lohmann H, Buck-Gramcko D, Martin L. Pseudarthrosis of the scaphoid bone: experiences in 240 cases. Handchir Mikrochir Plast Chir 1987;19:3069 .

31. Radford PJ, Matthewson MH, Meggitt BF. The Herbert screw for delayed and non-union of scaphoid fractures. J Hand Surg [Br] 1990;15:455-9.

32. Stark HH, Rickard TA, Zemel NP, Ashworth CR. Treatment of ununited fractures of the scaphoid by iliac bone grafts and Kirschner-wire fixation. J Bone Joint Surg [Am] 1988;70-A:982-91

33. Moran R, Curtin J. Scaphoid fractures treated by Herbert screw fixation. J Hand Surg [Br] 1988;13:453-5.

34. Fernandez DL. Anterior bone grafting and conventional lag screw to treat scaphoid non-unions. J Hand Surg [Am] 1990;15:140-7.

35. Prosser GH, Isbister ES. The presentation of scaphoid non-union. Injury 2003;34:65-7.

36. Herzberg G, Forissier D, Falaise C. Coronal fractures of the proximal scaphoid: the proximal ring sign. J Hand Surg [Br]2003;28:500-3. 